\title{
Matching atomistic simulations and in situ experiments to investigate the mechanics of nanoscale contact.
}

\author{
$1 *$ Sai Bharadwaj Vishnubhotla, ${ }^{2 *}$ Rimei Chen, ${ }^{1}$ Subarna R. Khanal, ${ }^{2}$ Xiaoli Hu, ${ }^{2}$ Ashlie \\ Martini, ${ }^{1}$ Tevis D. B. Jacobs \\ * indicates equal contribution. \\ 1. Department of Mechanical Engineering and Materials Science, University of Pittsburgh, \\ Pittsburgh, PA, USA. \\ 2. Department of Mechanical Engineering, University of California-Merced, Merced, CA, USA.
}

The published version of this article can be found at: doi: 10.1007/ s11249-019-1210-7

Full citation information is as follows:

Vishnubhotla, S.B., Chen, R., Khanal, S.R., Hu, X., Martini, A., Jacobs, T.D.B.:

Matching Atomistic Simulations and In Situ Experiments to Investigate the Mechanics of Nanoscale Contact Tribology Letters 67, 97 (2019).

\begin{abstract}
$\underline{\text { Abstract }}$
Many emerging devices and technologies rely on contacts between nanoscale bodies. Recent analytical theories, experiments, and simulations of nanocontacts have made conflicting predictions about the mechanical response as these contacts are loaded and separated. The present investigation combined in situ transmission electron microscopy (TEM) and molecular dynamics (MD) simulation to study the contact between a flat diamond indenter and a nanoscale silicon tip. The TEM was used to pre-characterize the materials, such that an atomistic model tip could be created with identically matched materials, geometry, crystallographic orientation, loading conditions, and degree of amorphization. A large work of adhesion was measured in the experiment and attributed to unpassivated surfaces and a large compressive stress applied before separation, resulting in covalent bonding across the interface. The simulations modeled atomic interactions across the interface using a Buckingham potential in order to reproduce the experimental work of adhesion without explicitly modeling covalent bonds, thereby enabling larger time- and length-scale simulations than would be achievable with a reactive potential. Then,
\end{abstract}


the experimental and simulation tips were loaded under similar conditions with real-time measurement of contact area and deformation, yielding three primary findings. First, the results demonstrated that significant variation in the value of contact area can be obtained from simulations, depending on the technique used to determine it. Therefore, care is required in comparing measured values of contact area between simulations and experiments. Second, the contact area and deformation demonstrated significant hysteresis, with larger values measured upon unloading as compared to loading. Therefore, continuum predictions, in the form of a Maugis-Dugdale contact model, could not be fit to full loading/unloading curves. Third, the loaddependent contact area could be accurately fit by allowing the work of adhesion in the continuum model to increase with applied force from 1.3 to $4.3 \mathrm{~J} / \mathrm{m}^{2}$. The most common mechanisms for hysteretic behavior - which are viscoelasticity, capillary interactions, and plasticity - can be ruled out using the TEM and atomistic characterization. Stress-dependent formation of covalent bonds is suggested as a physical mechanism to describe these findings, which is qualitatively consistent with trends in the areal density of in-contact atoms as measured in the simulation. The implications

of these results for real-world nanoscale contacts are that significant hysteresis may cause significant and unexpected deviations in contact size, even for nominally elastic contacts.

Keywords: Nanoscale contact, Adhesion, In situ TEM, Molecular dynamics simulation

\section{Introduction}

As devices and manufacturing technologies shrink to the nanoscale, there is a growing need to understand, predict, and control the behavior of small-scale contacting interfaces. Examples of this need stem from nanomanufacturing, materials characterization, and nanodevices. Specifically, in tip-based nanomanufacturing $[1,2]$ and in micro-transfer printing of quantum dots [3], the behavior of the nanocontact determines reliability and precision. In the vast array of scanning probe microscopy techniques $[4,5]$, the contact's size and properties determine both the resolution of the technique and the accuracy of models applied for quantitative analysis. Finally, for nanoscale switches and actuators $[6,7]$, the mechanical and transport properties of the contact determine functionality and lifetime of the device.

At present, it is common to use continuum mechanics theories to describe nanoscale contacts. These continuum theories predict the deformation, contact area, and adhesion of contacts under load. A thorough review of contact mechanics models can be found elsewhere [8]; only a brief summary is presented here. The Hertz model [9] relates deformation and contact area to applied load for two elastic spheres without adhesion. Adhesion was then considered in the DerjaguinMüller-Toporov (DMT) [10] and Johnson-Kendall-Roberts (JKR) [11] models to describe deformation and contact area. The DMT limit applies to stiff materials with weak and long-range adhesion, whereas the JKR limit applies to compliant materials with strong and short-range adhesion. First Tabor [12] and later Maugis [8] showed that the DMT and JKR are two extreme 
limits for describing a continuum of contact behavior. The transition region between the limits can be characterized by a dimensionless transition parameter $[8,12]\left(\mu_{T}\right.$ or $\left.\lambda\right)$ which is the ratio of elastic deformation to the length-scale of surface forces. In the transition region, Maugis [8] provided expressions for deformation and contact area as functions of applied load. These equations were simplified by Carpick et al [13] to present a numerical analysis for determining the contact area in the transition region. Similar numerical analysis was extended for determining deformation in the transition region [14].

These continuum models and their various extensions rely on underlying assumptions that may be violated at nanometer length scales. An alternative to continuum models is atomistic simulations, which are able to track the position of each atom and have been employed to study nanocontacts mimicking experimental conditions [15-19]. Some such simulations have suggested that contact mechanics may be applied when atomic-scale surface roughness is considered in the model [20] or when modifications of these theories are used, such as thin-coating contact mechanics [16, 21]. In particular, Milne et al. [22] showed that contact mechanics can be modified through the addition of a supplemental "force", which accounts for deviations from ideal geometry. This results in a supplemental work of adhesion term, which includes the effects of topography as well as the contribution to adhesion from covalent bonds at the interface, both of which can change during loading or sliding. However, other studies showed that continuum contact mechanics may break down at the atomic scale [23-27]. Further, due to the discreteness of atoms at the contact interface, the definition of contact is ambiguous at the atomic scale, which complicates comparison to continuum mechanics predictions [20, 28, 29]. A more in-depth treatment of these considerations can be found in Ref. [30].

Despite the reasons to doubt the nanoscale applicability of contact mechanics models, there are important reasons this topic requires further investigation. First, there are numerous atomic force microscopy (AFM) investigations where measured data have been accurately fit by applying continuum contact mechanics, as discussed in [13,31-33]. Second, the models are still very widely applied in both basic science (e.g., Ref. [34]) and engineering applications (e.g., Ref. [35, 36]) to compute contact properties. Third, it is impractical to rely on atomistic simulations in every contact situation because they are typically time- and resource-intensive and are often unique to a particular set of conditions and thus difficult to generalize. As such, there is benefit to having analytical expressions from contact mechanics models, even if they are approximate. The present paper combines the use of in situ transmission electron microscopy and molecular dynamics simulation to study the contact between a flat diamond indenter and a sharp silicon tip. The purpose of the present paper is to perform an in-depth analysis into a single contact experiment. The simulations were designed to faithfully reproduce the key features of the experiments so that the two can be used together to explore the adhesion, deformation, and contact area of the same nanoscale contact. 


\section{Methods}

\subsection{In situ TEM experimental measurements}

Contact tests were performed inside of a transmission electron microscope (2100F, JEOL, Tokyo, Japan) operated at $200 \mathrm{keV}$ with the use of an in situ nanoindenter (PI-95 Picoindenter, Hysitron Inc., Minneapolis, MN). The experimental setup is similar to that of [37-39] and is shown in Fig. 1 $(a, b)$. The tests were conducted by bringing a flat diamond indenter into contact with an AFM probe (PPP-NCLR, Nanosensors, Neuchatel, Switzerland) with real-time TEM video, as shown in Supplementary Movie 1. In contrast to prior in situ TEM investigations of tip/sample contact, the tests were performed under closed-loop displacement control using a capacitive sensor with a feedback rate of $78 \mathrm{kHz}$. The loading/unloading rate was $1.0 \mathrm{~nm} / \mathrm{s}$, and the real-time applied force and displacement were saved at 200 data points per second. High-resolution TEM images of the silicon tip were taken before and after the test to enable the measurement of geometry using image processing. Lower-resolution video was captured during the loading/unloading.

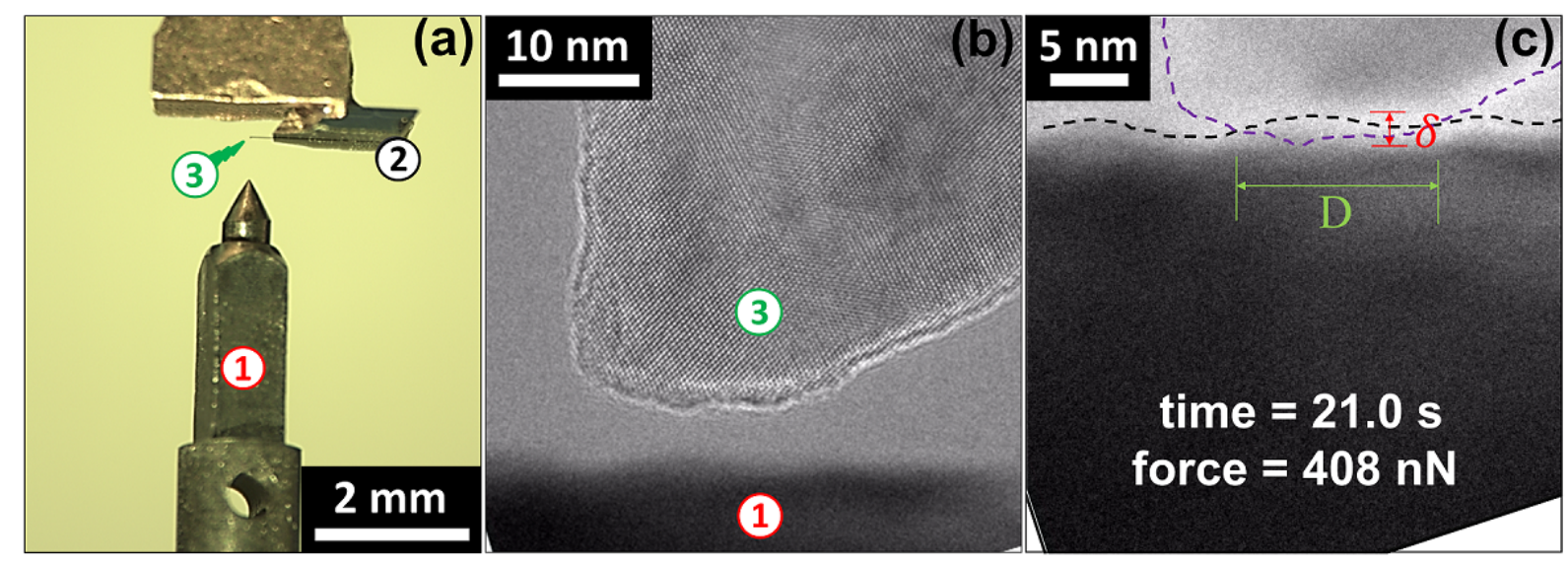

Figure 1. Optical (a) and TEM (b) images of the experimental setup enabling an in situ contact test inside of a transmission electron microscope. A flat diamond indenter (1) is opposite an AFM chip (2), such that it can be brought into contact with the nanoscale tip (3). During contact, the video frames (c) are used to measure real-time deformation and contact area.

Before testing, the diamond indenter was cleaned with light mechanical abrasion using a cotton swab and acetone. Next, the indenter was sequentially ultrasonicated in isopropanol, acetone, and methanol. This cleaning procedure is recommended by the manufacturer for nanoindentation tips, and has been used in earlier studies (for example [40]). Further, direct inspection using the TEM verified that there was no significant debris or contamination on the surface. This direct inspection was also used to rule out the possibility of carbonaceous contamination during imaging. While the build-up of carbonaceous contamination has been observed in electron microscopy [41], this is most problematic for very high electron doses and/or poor vacuum in the chamber. It was not observed in the present study, even after significant additional beam exposure after completion of the test. 
The AFM chip body was cleaved and glued to the sample mount of the nanoindenter. The commercial silicon AFM probe had an initial radius of approximately $5 \mathrm{~nm}$, and contained an oxide from the manufacturer's processing that extended to a height of $14 \mathrm{~nm}$ on the tip. This oxide was mechanically removed by bringing the indenter into contact with the tip and sliding it in vacuum prior to testing. During the mechanical abrasion, sufficient material was removed that the tip was shortened by $62 \mathrm{~nm}$, confirming the removal of all of the pre-existing oxide. At this point, the crystal lattice of silicon was observed to extend to the apex of the tip. The tip was then preloaded seven times to similar or larger stress levels than were planned for the present investigation. These pre-loading events were conducted to ensure that any inelastic deformation or change in small-scale roughness would occur prior to the analyzed test. Indeed, the pre-loading resulted in amorphization of the outermost $1.2 \mathrm{~nm}$ of the tip apex, as seen in Fig. 1b. This amorphization effect has been observed previously [42], and is often attributed to a transition under load to a metastable $\beta$-tin structure. The tip remained in the vacuum environment of the TEM throughout the investigation, such that the newly exposed silicon was not re-oxidized.

Following tip preparation, a loading and adhesion test was conducted and analyzed in depth. For this case, the contact diameter $D$ and deformation $\delta$ were measured directly from the experiments, as shown in Fig. 1c. Because of vibration in the contact and Fresnel fringes [41] around the largerdiamond indenter, the resolution of the in-contact videos are lower than the out-of-contact images. Thus, it was necessary to adjust the measured diameter using a correction factor. First, the high resolution TEM image (Fig. 1b) out-of-contact profile was traced. Second, the in-contact profile was traced using the reduced-resolution in-contact TEM video frame (Fig. 1c). The mean of the difference between the width of the in-contact and out-of-contact profiles was measured to be $0.3 \mathrm{~nm}$ and set as the correction factor. Hence, the corrected contact diameter was calculated as $D_{\text {true }}=D_{\text {meas }}-0.3 \mathrm{~nm}$. Finally, the contact was assumed to be circular, such that the contact area $A$ was $\pi D_{\text {true }}^{2} / 4$; this approach to calculating contact area is referred to subsequently as the "sideview diameter" method.

\subsection{Simulation methods}

The atomistic model of the nanocontact experiment is shown in Fig. 2. The model consisted of a silicon tip and a rigid substrate. According to the TEM imaging, the tip was composed of crystalline silicon with an amorphous region at the perimeter of its apex. The high-resolution TEM image of the tip in Fig. 2a was traced to extract the 2D profiles of both the crystalline and amorphous regions. The method of disks [37] was used to create 3D surfaces for the tip shape, under the assumption that the $2 \mathrm{D}$ profile is representative of the tip shape in all orientations. This assumption is supported by previous work on sliding wear of silicon. For example, Ref. [43] compared side-view TEM images to the results of numerical 3D tip reconstruction from AFM scans; the results showed similar radii in all orientations. Further, using calculations for bodies of dissimilar parabolic radii in different directions [44], it can be shown that even a difference of $5 \%$ 
in tip radii between in-plane and out-of-plane orientations causes an error of less than $1 \%$ in computed results.

The crystallographic direction of the crystalline region of the tip was set based on the diffraction pattern from the TEM. The height of the tip model was $10 \mathrm{~nm}$. The substrate consisted of carbon atoms in a diamond lattice and had dimensions of $20 \times 20 \times 1 \mathrm{~nm}$ in the $x, y$, and $z$ directions respectively. The substrate atoms were held rigid for computational efficiency. According to the Hertz model, this assumption is expected to introduce differences in contact area and deformation of approximately $7 \%$ from the experiment. However, this was accepted since an accurate description of elasticity on both bodies would have required a significant increase in the simulation size. The substrate was modeled as a flat surface because the radius of curvature of the overall shape of the indenter was measured to be $1 \mu \mathrm{m}$. Nanoscale roughness on the substrate was neglected because its accurate inclusion would require knowledge of the precise location of the tip contact - including in the out-of-plane direction - which cannot be identified with sufficient precision due to the finite resolution in the TEM. Fixed boundary conditions were applied in all directions.

The topmost $1 \mathrm{~nm}$ of the tip was treated as rigid. A Nosé-Hoover thermostat was applied to the 4-nm region immediately below the rigid layer to control the temperature of the system at $300 \mathrm{~K}$. The remainder of the tip atoms were integrated using the NVE (constant number of atoms, volume, and energy) ensemble, which enabled the simulation to capture the dynamics of atoms in the contact interface [15]. Simulations were carried out using the molecular dynamics simulation package LAMMPS [45] with a time step of 1 fs.

There were four processes during the simulation: equilibration, loading, relaxation, and unloading. In each process, the interaction force between the tip and substrate was characterized. The interaction force was calculated as the sum of the forces on the tip atoms [27]. The tip was initially placed at a distance of $0.7 \mathrm{~nm}$ above the substrate. This distance was greater than the cut-off distance of the empirical potentials. Thus, there was no interaction between the tip and the substrate during the equilibration process. After the system reached stability in temperature and energy, which indicated the system was at equilibrium, a constant downward velocity of $5 \mathrm{~m} / \mathrm{s}$ was applied to the rigid portion of the tip. The tip moved towards the substrate until it reached the maximum force observed from experiment. After the tip reached the maximum force, it was relaxed for $0.7 \mathrm{~ns}$ to ensure stability in energy and force. After relaxation, the tip was retracted from the substrate at $5 \mathrm{~m} / \mathrm{s}$ to simulate an unloading process. Due to realistic limitations of computation time, the loading and unloading speeds were significantly faster than experiment. However, contact mechanics models do not predict an effect of pull-off speed for hard, non-viscoelastic materials. Further, long-timescale processes such as creep are not expected to play a significant role in the present contact. To verify this, we repeated the simulations at speeds ranging from 0.2 to $10 \mathrm{~m} / \mathrm{s}$ and observed no statistically significant effect on pull-off force. 
The modified Tersoff potential [46], which is known to be able to accurately capture the mechanical properties of silicon, was employed to simulate the interactions within the silicon tip. The interactions between tip and substrate were modeled by the Lennard-Jones potential with the addition of a Buckingham potential to capture the large short-range adhesive interactions that were observed in the experiment. The determination of interaction parameters is discussed in Sect. 3.1. The deformation of the tip in the simulation was determined by subtracting the sum of the initial heights of the tip and substrate from the instantaneous distance between the top of the tip's rigid layers and the bottom of the substrate. This results in a value that is consistent with the continuum definition of deformation. Contact area in the simulation is calculated as described in Sect. 3.3.
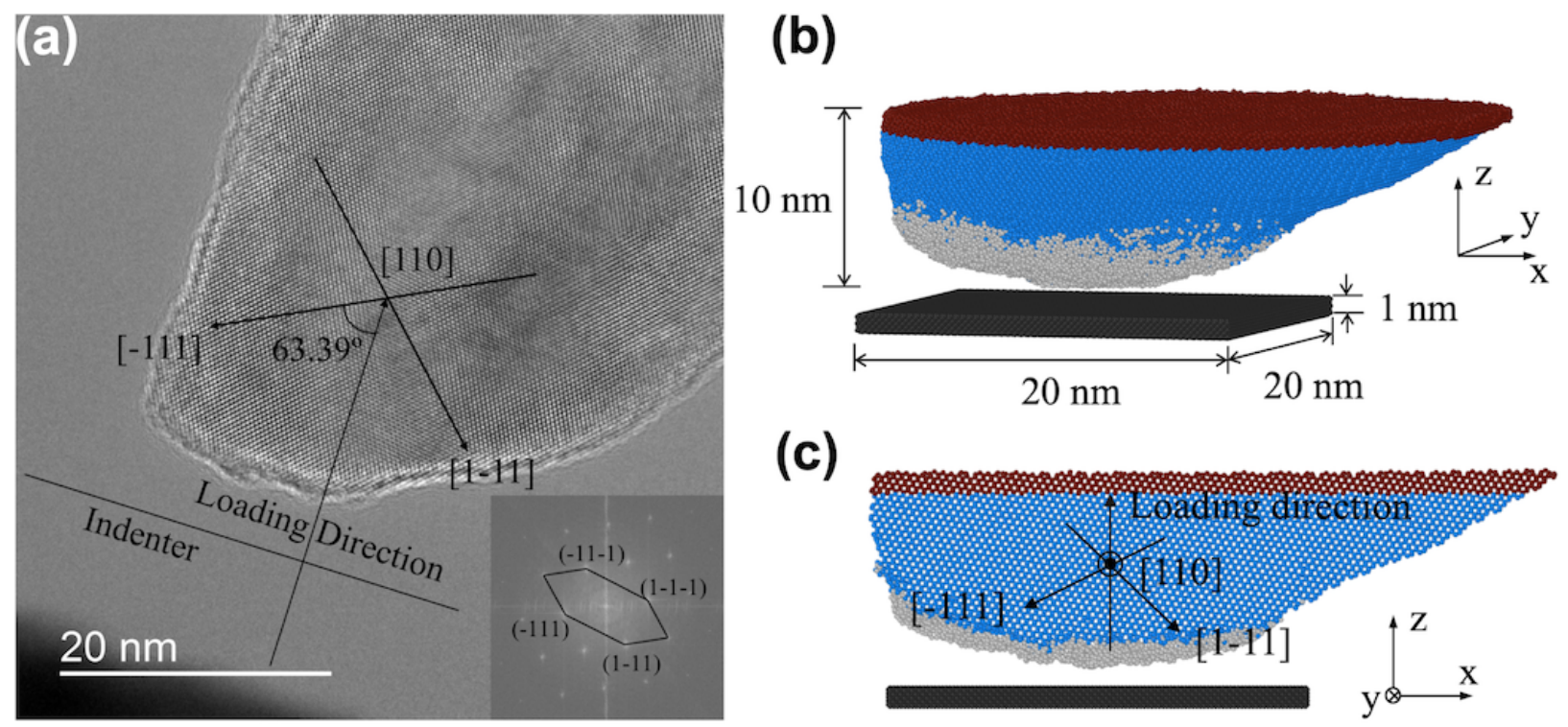

Figure 2. A high-resolution TEM image (a) of the AFM tip is shown with crystallographic and loading directions labeled. The inset image shows the 2-D Fourier transform of the corresponding TEM image. The atomistic model is shown in perspective (b) and cross-sectional (c) views. Sphere color represents atom type, where: blue is crystalline silicon; silver is amorphous silicon; red is the rigid part of the tip; and black is carbon.

\section{Results and Discussion}

\subsection{Matching adhesive interactions between simulations and experiments}

To ensure that the simulations and experiments were describing the same nanocontact, it was necessary to match the adhesion strength at the interface, as quantified by the pull-off force. The force-vs-time data from experiment is shown in Fig. 3a where the maximum displacement of the AFM probe corresponds to the maximum force of $408 \pm 31 \mathrm{nN}$ achieved in the test. During unloading, the pull-off event can be observed visually in the TEM video (Supplementary Movie 1). The pull-off force is the amount of force required to break the contact; in this case it was found to be $434 \pm 31 \mathrm{nN}$. 
In the simulations, the adhesive interactions between the atoms in the two surfaces were described using the sum of the Lennard-Jones and Buckingham forces. The Lennard-Jones potential approximated the van der Waals attraction and Pauli repulsion between the materials, and the parameters were set as $\varepsilon=0.0024 \mathrm{eV}, \sigma=0.28 \mathrm{~nm}$ [40]. The relatively large adhesion observed in the experiment was likely due to the formation and breaking of covalent bonds during the test. Covalent bonding could be modeled explicitly in the simulation using a reactive potential, but this approach would severely limit the size of the system. Therefore, an approximation was introduced that captured the adhesion in an effective way for a sufficiently large model system. Specifically, the Buckingham potential was used to add an additional attractive force between the tip and the substrate $[47,48]$. The Buckingham potential has the form $E=-\alpha e^{-r / \zeta}$, where $\alpha$ is the maximum attractive energy between two atoms, and $\zeta$ is the characteristic short-range decay length. The value of $\zeta$ was set to be 0.3 times the value of $\sigma$ of the Lennard-Jones potential $[47,48]$. The pulloff force increased approximately linearly with the magnitude of the parameter $\alpha$, and the experimental pull-off force was reproduced in the simulation using a value of $\alpha=3.5 \mathrm{eV}$. This extracted Buckingham parameter $\alpha$ is comparable to the energy of a silicon-carbon bond, which is $3.3 \mathrm{eV}$ [49]. The simulation force-vs-time data is shown in Fig. 3b. The maximum force and the pull-off force in the simulation were $367 \pm 2 \mathrm{nN}$ and $457 \pm 8 \mathrm{nN}$, respectively.
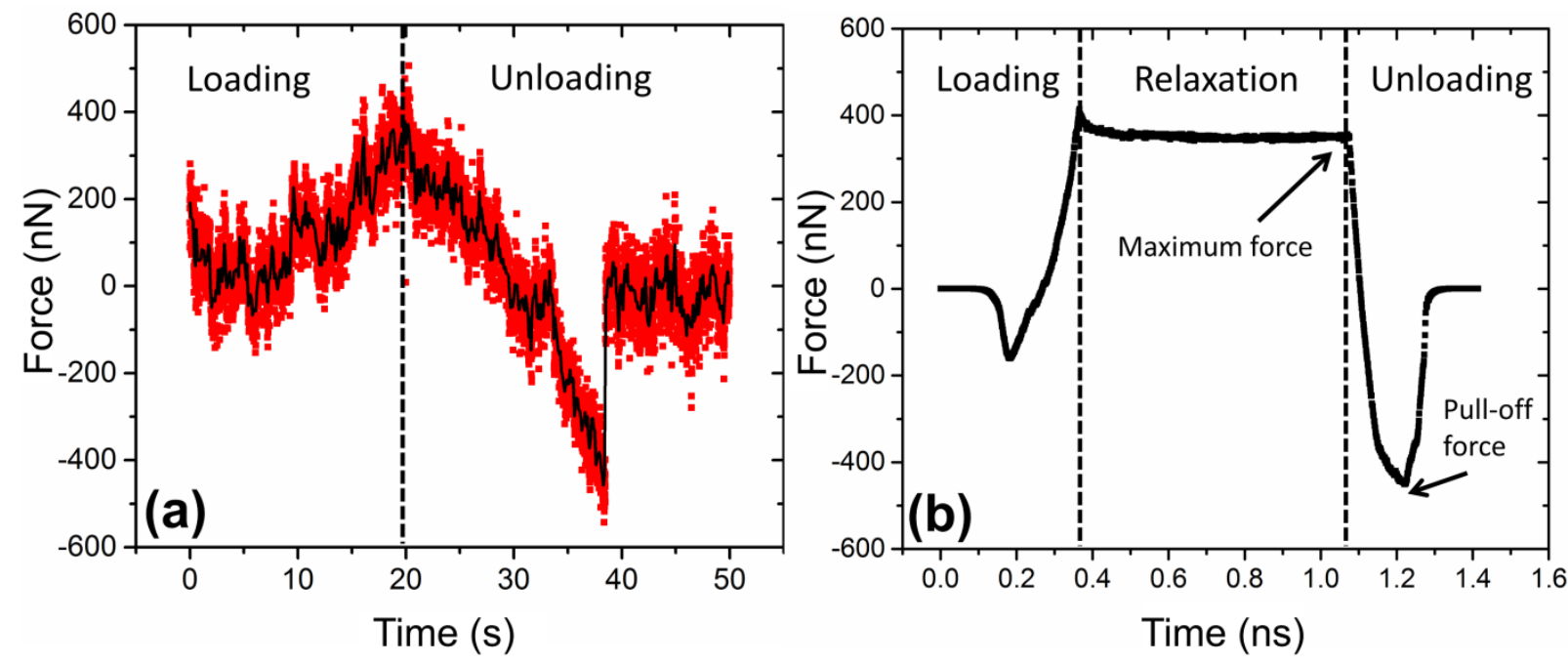

Figure 3. The force as a function of time from experiment (a), shown as both raw (red symbols) and downsampled (black line) data, and from simulation (b). As shown, the maximum and pull off forces are matched between experiment and simulation, while the timescales differ significantly.

\subsection{Measuring work of adhesion from the experiments}

To determine the work of adhesion from the experiments, the method of Ref. [50] was used. Specifically, three steps were performed: (1) evaluate the geometry of the body to confirm a parabolic shape and measure tip radius; (2) compare the shapes of the bodies before and after contact to confirm that there was no change in overall shape due to testing; and (3) determine 
which of the continuum contact models is predicted to apply by computing the Tabor parameter for the contact.

To evaluate the geometry of the bodies, the outer contour of the tip was traced using image analysis. To verify near-parabolic shape (Step 1), the traced contour was fit to a parabola of the form $z=x^{2} / 2 R$, where $z$ is the vertical height, $x$ is the lateral dimension, and $R$ is the radius. To assess changes with testing (Step 2), the traced contours were compared before and after testing. Before testing, the tip radius was $20.0 \pm 1.8 \mathrm{~nm}$ (Fig. 4a) and the root-mean-square (RMS) deviation from the paraboloidal shape was $0.17 \mathrm{~nm}$. After testing, the tip radius was $20.7 \pm 1.4 \mathrm{~nm}$ (Fig. 4b) with an RMS deviation of $0.16 \mathrm{~nm}$. While there were Angstrom-scale modifications to the tip, the tip remained paraboloidal and the radii were indistinguishable within experimental uncertainty. The geometric assessment supports the application of classical contact mechanics models.
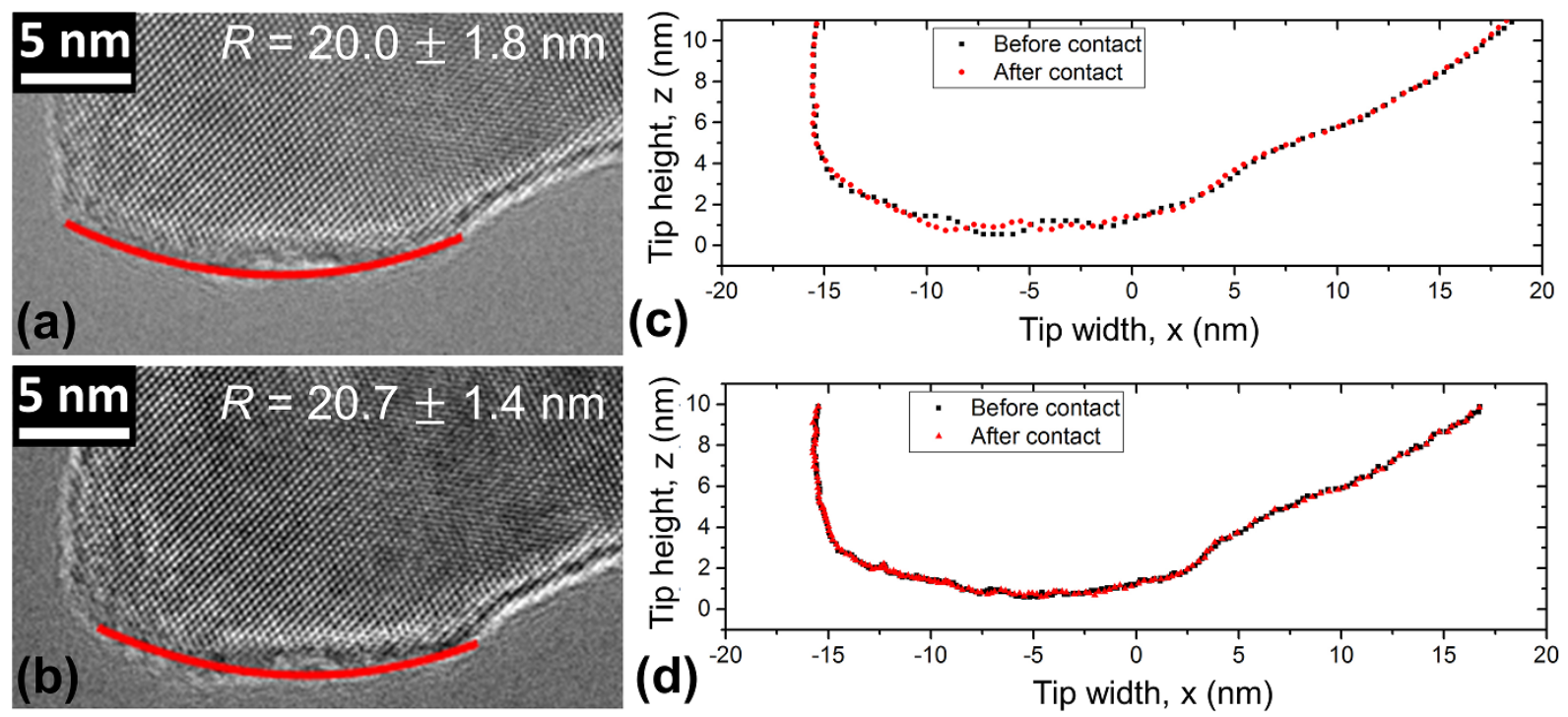

Figure 4. TEM images of the tip (a) before and (b) after contact with best fit parabolas shown in red. Tip profiles are compared in the experiment (c) before and after contact and in the simulation (d) before and after contact. These results confirm a lack of permanent shape change due to plasticity or fracture.

To determine which of the contact models is predicted to apply (Step 3), the Tabor parameter [12] was computed [50]. This required first obtaining the work of adhesion, which was calculated from continuum contact mechanics as follows [13]:

$$
W_{a d h}=\frac{F_{p u l l-o f f}}{\chi \pi R}
$$

where $F_{\text {pull-off }}$ is the pull-off force, $R$ is the asperity radius, and $\chi$ is a constant that is equal to 1.5 for the JKR model, 2 for the DMT model, and an intermediate value for Maugis-Dugdale model. Using this equation and measured parameters, the work of adhesion computed for the 
diamond and silicon pair was found to be in the range of $3.3 \pm 0.2 \mathrm{~J} / \mathrm{m}^{2}$ (DMT) and $4.4 \pm 0.3 \mathrm{~J} / \mathrm{m}^{2}$ (JKR). Using the procedure described in Ref. [13, 50], with a range of adhesion of $0.2-0.3 \mathrm{~nm}[32,40,50-52]$, the Tabor parameter [12] was found to be $\mu_{T}=0.8-1.5$ and the work of adhesion was found to be $4.2 \pm 0.4 \mathrm{~J} / \mathrm{m}^{2}$. This indicates that the contact lies in the transition region between the JKR and DMT limits, and should be described using the MaugisDugdale model [8]. Here, this model was numerically implemented using the Carpick-OgletreeSalmeron (COS) method [13].

The measured work of adhesion was larger than previous measurements. While prior ex situ testing of diamond/diamond, silicon/silicon, and diamond/silicon contacts have yielded $W_{\text {adh }}=0.1-0.7 \mathrm{~J} / \mathrm{m}^{2}$ [40, 43, 53-56], prior in situ TEM testing [22] of a silicon/diamond contact demonstrated significantly higher work of adhesion values, due to bonding across the interface and changing interfacial roughness, both of which are modified by loading of the contact. The work of adhesion for an ideally bonded contact can be computed [57] as the product of the areal density of bonds and the bond energy. Using minimum and maximum surface bond densities of approximately $6.8 \times 10^{18}$ atoms $/ \mathrm{m}^{2}$ and $9.6 \times 10^{18}$ atoms $/ \mathrm{m}^{2}$ (calculated from the surface atom density of silicon in the orientations [ $\left.\begin{array}{llll}1 & 0 & 0\end{array}\right]$ and [ 1100$]$ respectively) and a bond energy of $318.0 \mathrm{~kJ} / \mathrm{mol}$ [49], the covalent work of adhesion is $3.6-5.1 \mathrm{~J} / \mathrm{m}^{2}$ between silicon and diamond. Therefore, the measured work of adhesion is commensurate with covalent bonding across the interface.

\subsection{Contact area and variations with calculation technique}

To demonstrate the variation in contact area calculated from the simulations, the area of contact at the maximum force was evaluated using a variety of different methods. These methods are discussed in more detail in Ref. [30].

In the simulation, contact area was obtained by first identifying the atoms that are "in contact", which was done using two different criteria: (1) distance; and (2) force. In the distance criterion, if the distance between a tip atom and its nearest substrate atom was less than a pre-defined limit $d_{c}$, then this tip atom was considered to be in contact with the substrate. The value of $d_{c}=0.25 \mathrm{~nm}$ was chosen as it is the distance at which the $\mathrm{Si}-\mathrm{C}$ interatomic potential energy is a minimum [20, 29, 58]. In the force criterion, the time-averaged interaction force was calculated between each tip atom and the substrate. If the tip atom experienced a repulsive force from the substrate, this tip atom was considered to be in contact with the substrate $[59,60]$. The time-averaged forces on the tip atoms were calculated over different time intervals at steady state, i.e., the observation time interval varied from 1 to 10,000 time steps. The results from both methods are shown in Fig. 5 for the point of maximum force.

Using the force criterion, the number of contact atoms increased with increasing force-averaging time interval and then saturated after approximately 1000 time steps. This time-dependence does 
not reflect physical changes in the material, but is rather attributed to thermal fluctuations and the fact that short-time fluctuations are averaged out $[59,60]$. Therefore, only the longest observation period is reported in the rest of the present discussion, in accordance with Ref. [60]. Using the force criterion with the longest observation period yielded a value of $1383 \pm 4$ atoms. Using the distance criterion, the mean number of contact atoms was $1672 \pm 9$. This result is shown as a horizontal bar in Fig. 5a, where the width of the bar represents the standard deviation of the data. Although results from both the force and distance criteria calculations are shown in Fig. 5a, the distance criterion results are inherently time-averaged and so do not depend on observation time.

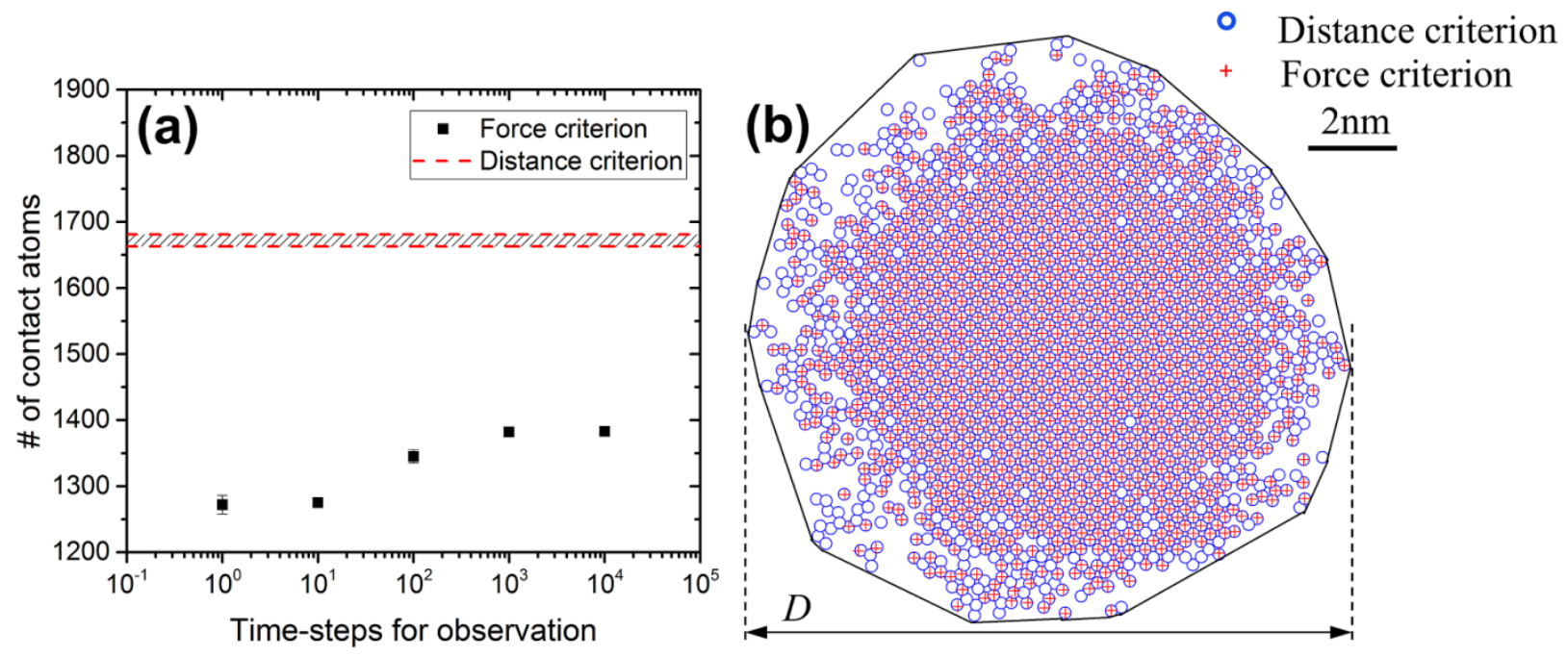

Figure 5. (a) Number of contact atoms at the maximum force as calculated using the distance criteria and the force criteria with increasing observation time. (b) Representative image of the contact atoms identified from the distance criterion (blue circles) and the force criterion (red crosses). The convex hull method defines the contact area enclosing the contact atoms (black outline). The side-view diameter method defines a circular contact with a diameter measured along the axis corresponding to the viewing direction of the TEM (marked as $D$ ).

Then, the number of atoms was converted into a measurement of contact area using each of three different methods: (1) multiplying the number of contact atoms by the area of a single atom (called "atomic area method"); (2) measuring the area of a polygonal region enclosing the contact atoms (called "convex hull method"); and (3) computing the area of a circular contact described by the diameter that is measured in an orientation that corresponds to the viewing direction of the TEM (called "side-view diameter method"). These methods are illustrated in Fig. 5b. As expected, the atomic area method yielded the smallest estimated area $\left(73.1 \pm 0.4 \mathrm{~nm}^{2}\right.$ and $58.8 \pm 0.4 \mathrm{~nm}^{2}$ for the distance and force criterion, respectively). The area from the convex hull method was significantly larger $\left(131.3 \pm 0.8 \mathrm{~nm}^{2}\right.$ and $125.6 \pm 1.0 \mathrm{~nm}^{2}$ for the distance and force criterion, respectively) because this calculation assumes continuous contact [20, 28]. Lastly, the side-view diameter method, which is most similar to the area obtained from TEM images in the experiment, 
resulted in the largest estimated area $\left(138.5 \pm 2.8 \mathrm{~nm}^{2}\right.$ and $132.8 \pm 0.2 \mathrm{~nm}^{2}$ for the distance and force criterion, respectively).

For comparison, at the maximum force, the contact area obtained from the TEM image (Fig. 1c) was $149.8 \pm 55.4 \mathrm{~nm}^{2}$, where the uncertainty is due to vibration of the indenter. The DMT, JKR and the Maugis-Dugdale theories predict values of $69.1 \pm 4.7 \mathrm{~nm}^{2}, 142.2 \pm 9.6 \mathrm{~nm}^{2}$, and $122.8 \pm 7.6 \mathrm{~nm}^{2}$, respectively. These are using the radii and works of adhesion as described in Sect. 3.2, and an effective modulus of $126.9 \mathrm{GPa}$. This effective modulus was computed as $E_{\text {eff }}=$ $\left[\frac{\left(1-v_{1}^{2}\right)}{E_{1}}+\frac{\left(1-v_{2}^{2}\right)}{E_{2}}\right]^{-1}$, where the first material is [ $\left.\begin{array}{lll}1 & 0 & 0\end{array}\right]$ diamond $(\mathrm{E}=1050.0 \mathrm{GPa}, v=0.1)[61]$ and the second material is silicon, oriented along the loading direction. The latter properties were $\mathrm{E}=132.8 \mathrm{GPa}$ and $v=0.28$, which were obtained from elastic constants $\mathrm{C}_{11}=165.6 \mathrm{GPa}$, $\mathrm{C}_{12}=63.9 \mathrm{GPa}, \mathrm{C}_{44}=79.5 \mathrm{GPa}$ [62] and a loading direction of [2 $\left.\overline{2} 19\right]$.

The results of all contact area calculations are shown in Fig. 6. The variation in contact area shown for each of the three continuum mechanics models reflects the uncertainty in the radius fit to the pre-test TEM images of the tip. Variation in the side-view diameter contact area corresponds to the resolution of the in-contact TEM images. Comparison of all approaches and the uncertainly associated with each demonstrates the wide range in possible measured values, even for a simulated contact with known atomic positions. This underscores the complexity of defining an accurate value for contact area and the care that is required to do so. It is likely that the "correct" area of contact to use will depend on the intended application. For instance, the rate of atomicscale wear via chemical reactions or the contact conductance for ballistic electron transport [63] will likely depend more strongly on the atomic area of contact. While the electrical transport in the diffusive limit will likely depend more strongly on the size of the contact patch, similar to the side-view diameter. For the remainder of this discussion, the side-view diameter from the TEM experiments will be compared against the side-view diameter method from the simulation, as these are the most directly analogous. Overall, the variability in computed values demonstrates that caution is required whenever the absolute value of contact area is being computed. For a more robust analysis, the contact area must be determined at a variety of applied forces, as is done in the next sub-section. 


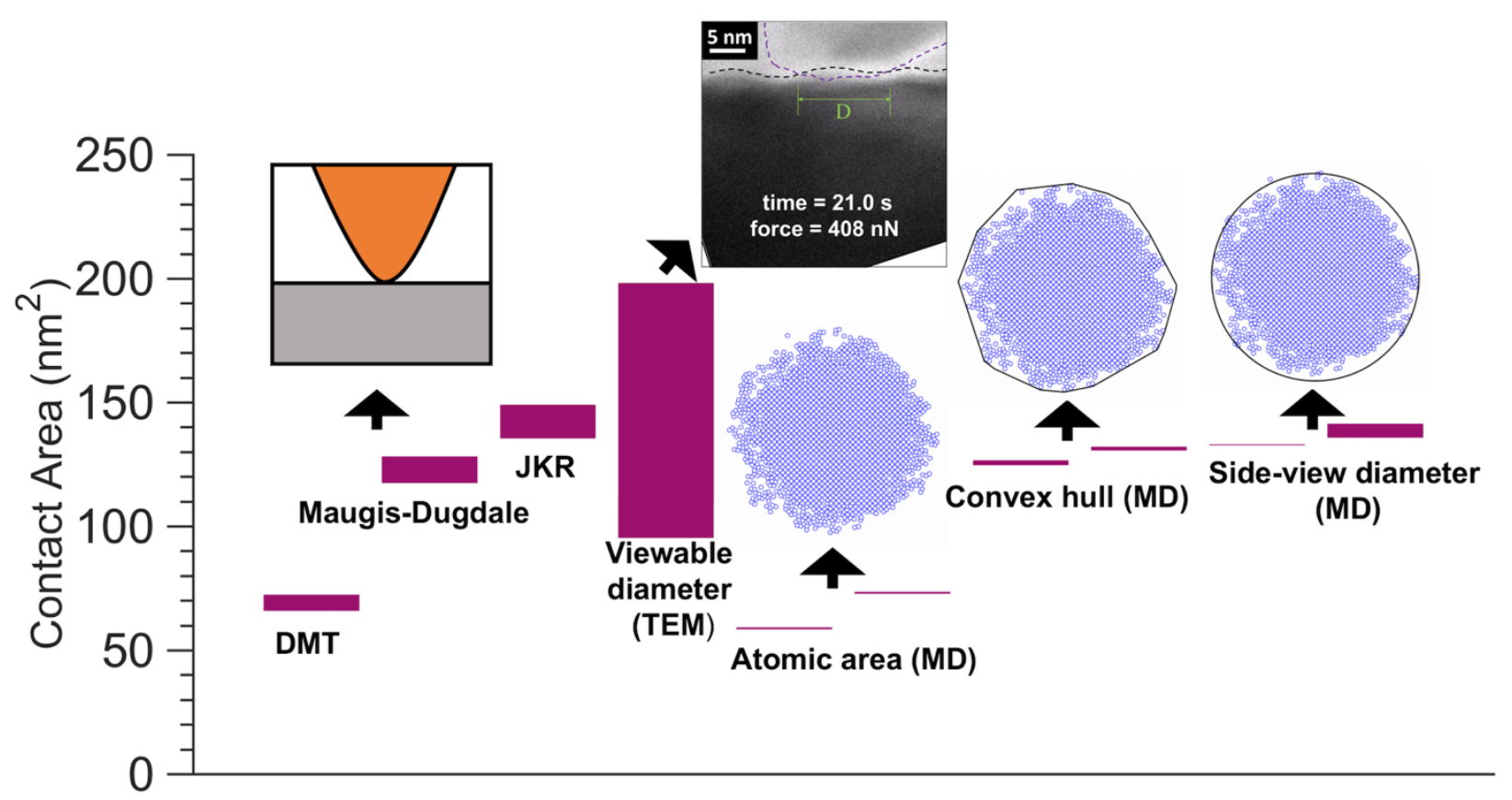

Figure 6. Contact area at the maximum applied force of $408 \mathrm{nN}$ : as predicted by continuum contact mechanics theories; as obtained from the in situ high-resolution TEM video; and as calculated from the atomistic simulations using different approaches. For each of the simulation measurements, two values are shown corresponding to the force (left) and distance (right) criteria.

\subsection{Contact area and deformation as a function of force.}

The contact area and deformation as functions of applied force are shown in Fig. 7. Experimental and simulated measurements for the area of contact agree within the uncertainty of the measurement. The uncertainty of the experimentally measured values arose due to vibration of the in situ indenter. For deformation, the experiments and simulations agree for the adhesive region only, and differ by more than experimental uncertainty for the compressive region. This difference at high loads may arise due to the finite size of the simulated tip, where the presence of the rigid layer artificially stiffens the contact. 


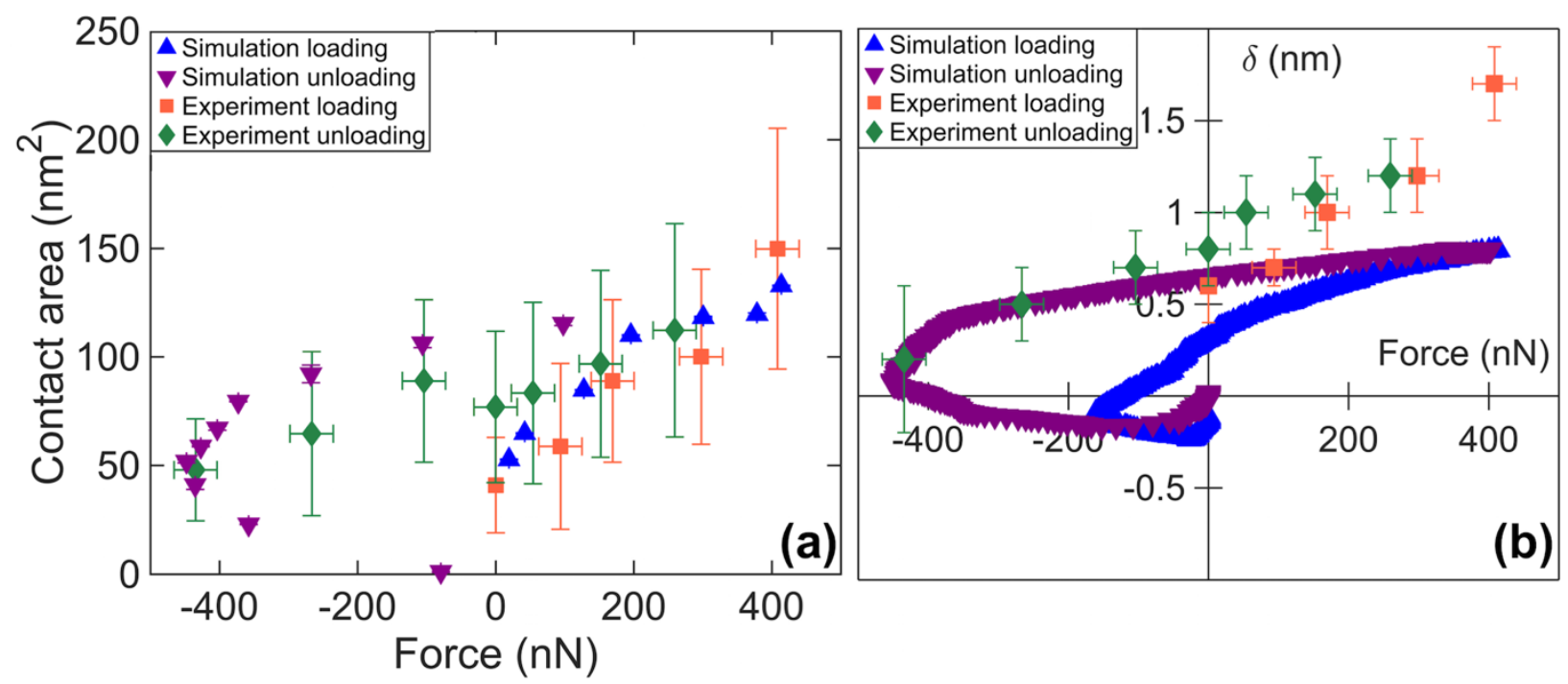

Figure 7. Contact area (a) and deformation (b) as a function of applied normal force, from experiment (squares) and simulation (triangles).

Both the experiments and simulations demonstrate hysteresis in behavior between the loading and unloading portions of the tests. The contact area at zero applied force is larger upon unloading by $88 \%$ (in experiments) and $110 \%$ (in simulations) as compared to the same value measured during loading. The difference for the deformation is $33 \%$ for the experiments and $109 \%$ for the simulations. This hysteresis behavior is not predicted by continuum elastic models. While this can be a hallmark of permanent changes within the material, there is no gross shape change observed before/after testing, as discussed in the previous section.

\subsection{Quantifying the hysteresis in contact area: Increasing work of adhesion as a function of maximum stress.}

None of the contact mechanics models (JKR, DMT, Maugis-Dugdale) could capture the hysteresis between loading and unloading behavior; therefore these two segments were investigated independently. The simulated unloading curve could be accurately fit using the intermediate case of Maugis-Dugdale with an effective modulus of $96.8 \mathrm{GPa}$ (free parameter) for a transition parameter $\lambda=1.6$ and work of adhesion of $4.4 \mathrm{~J} / \mathrm{m}^{2}$ ( $\lambda$ and $W_{\text {adh }}$ are simultaneously solved from the measured pull-off force and the best-fit $E_{\text {eff }}$ using the numerical analysis provided by COS method [13]). The experimental unloading curve was accurately fit also using the Maugis-Dugdale theory, with $E_{\text {eff }}=126.0 \mathrm{GPa}$ (free parameter) for $\lambda=1.3$ and $W_{\text {adh }}=4.3 \mathrm{~J} / \mathrm{m}^{2}$. The above analysis assumed an equilibrium separation $z_{o}=2.5 \AA$. Repeating this analysis using values in the range of $2.0 \AA-3.0 \AA$ resulted in small changes of the best-fit effective modulus $( \pm 5.2 \%$ for experiment, and $\pm 5.7 \%$ for simulation). The extracted best-fit value for effective modulus from the experiment matches well with that of crystalline silicon, which is $126.9 \mathrm{GPa}$ as computed in a previous subsection. 
By contrast, the Maugis-Dugdale model with the same parameters overestimated the contact area upon loading by $51 \%$. Indeed, the loading portion of the curve could not be accurately fit for any value of $E_{\text {eff. }}$ Therefore, the fit was retried allowing for a variable work of adhesion. Specifically, $W_{a d h}$ was allowed to vary with force, while the radius was held constant at the measured value $(20.7 \mathrm{~nm})$ and the effective modulus was held constant at the best-fit value determined in the previous paragraph (111.4 GPa, the average of the experimental and simulated best-fit values). Figure 8a shows the measured data, alongside curves representing the Maugis-Dugdale model with varying works of adhesion. Figure $8 \mathrm{~b}$ shows the extracted best-fit work of adhesion at each value of the stress in the contact. This best-fit value increases monotonically from $1.3 \mathrm{~J} / \mathrm{m}^{2}$, which accurately fits the contact area and force at the initial point, to $4.3 \mathrm{~J} / \mathrm{m}^{2}$, which fits well the contact area and force for the unloading data.

The variation between properties measured during loading and unloading has been observed in a variety of materials and is often referred to as adhesion hysteresis [57]. This effect is traditionally attributed to capillary effects [64] or to viscoelasticity in soft materials [65, 66], or to plasticity in hard materials [67]. Because the present test was performed in a vacuum environment, using hard non-viscoelastic materials (silicon and diamond), capillarity and viscoelasticity can be ruled out. Further, unlike in traditional AFM testing, the in situ TEM images and the MD simulations were used to rule out significant shape change upon testing. Atomic-scale plasticity of the type shown in Ref. [22] was not observed, likely because no sliding was induced and the tip had been preloaded to higher loads before these experiments were conducted. Therefore, it is expected that inelastic deformation would have already taken place prior to the present test. Instead, in these silicon/diamond contacts, the origin of the adhesion hysteresis is understood to be covalent bond formation, as discussed in Ref. [22]. As described in that work, this is equivalent to using continuum contact mechanics with a modified $W_{a d h}$, which depends on loading conditions, including the amount of pre-load and speed of lateral sliding. The physical origin of these changes in $W_{a d h}$ are increased covalent bond activity from stress in the contact.
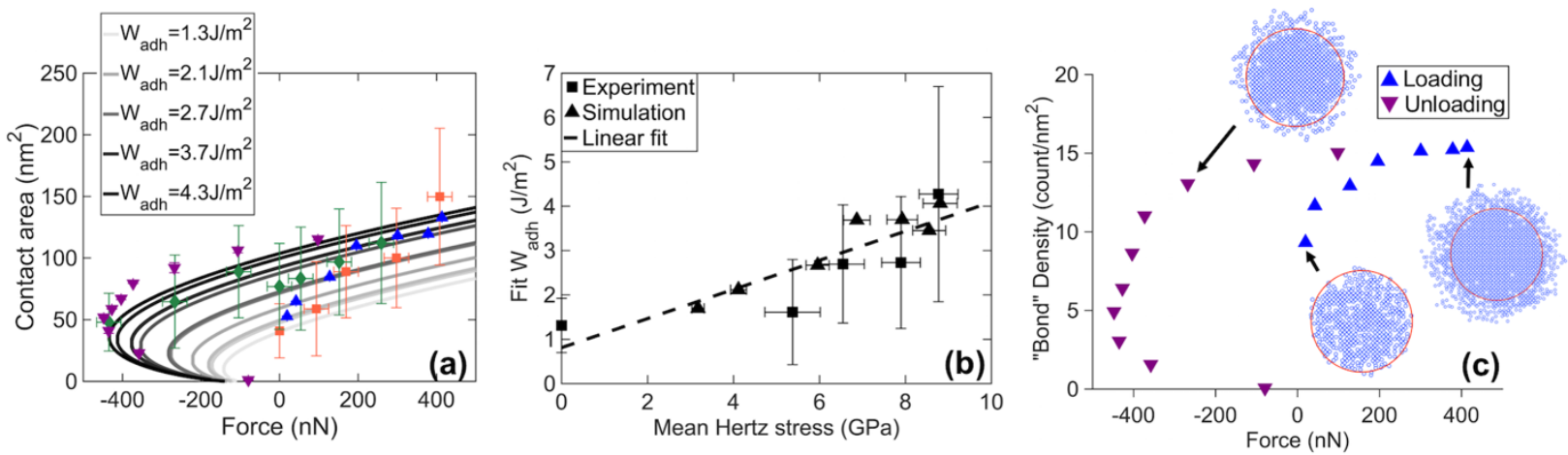

Figure 8. The data for contact area versus force (a) has been fit using the Maugis-Dugdale model with varying work of adhesion. The effective modulus was set using the best-fit value of $111.4 \mathrm{GPa}$ for unloading (see main text), and the work of adhesion is varied to separately match each individual point in the loading curve. The result of this point-by-point fit (b) shows a monotonically increasing value of 
work of adhesion with increasing mean Hertz stress. The meaning of the symbols in (a) are the same as described in Fig. 7. The simulation results were used to compute the areal density of in-contact atoms (c) in the central region of contact (red circle). The initial contact area shows a lower density of "bonds", which increases monotonically throughout loading. Notably, the high final "bond" density is maintained during unloading to large negative forces.

Further insight is obtained by combining the in situ experiments with the MD simulations to examine atomic-scale interactions in the contact interface. Specifically, a measurement was taken of the areal density of in-contact atoms, identified using the force criterion. This is loosely analogous to a bond density; however, in the absence of a reactive potential, the concept of a "bond" is not well-defined. For a fixed central region of the contact, this areal density was measured at various points throughout the test, and is shown in Fig. 8c. The result demonstrates a monotonic increase from the initial value to the value at the highest force. In other words, for a given area of contact, the additional loading has pushed a larger number of atoms into close contact and thus into the deepest part of the interatomic potential. Therefore, this region of contact will require more energy to separate, thus corresponding to a larger work of adhesion in a continuum description. Indeed, the high density of in-contact atoms achieved at the maximum force $(400 \mathrm{nN})$ was maintained (within 10\%) throughout unloading, all the way to the point where a tensile force of approximately $270 \mathrm{nN}$ was applied. Overall, the $65 \%$ increase in "bond" density during loading does not fully explain the measured increase in work of adhesion; however, the qualitative trends are similar. Therefore, the present results further elucidate the adhesion hysteresis that arises due to stress-dependent bond formation across the interface. Further simulations with a reactive potential are required to explore the details of the bond formation and breaking under applied force.

\section{Conclusions}

This investigation comprised a comprehensive analysis of the loading and separation of a nanocontact using experiments and simulations. The experiment and simulation were matched regarding materials, geometry, structure, crystal orientation, and applied forces. The Buckingham potential was used to model the interactions across the interface, and to reproduce the pull-off force observed in the in situ test. First, it was demonstrated that the choice of method for computing contact area can lead to significant variations in measured results. Second, the experimental and simulated measurements for contact area and deformation as a function of applied force demonstrated hysteretic behavior, with larger values measured upon unloading as compared to loading. Therefore, they could not be accurately fit using a straightforward application of continuum mechanics via the Maugis-Dugdale model. For contact area, the Maugis-Dugdale model could be accurately fit to the unloading portion of the contact area curve with a reasonable value of effective elastic modulus extracted from the fit; however, the same model over-predicted contact area by an average of $51 \%$ during loading. Third, a significantly better fit to contact area was found by allowing the work of adhesion to increase with applied force from 1.3 to $4.3 \mathrm{~J} / \mathrm{m}^{2}$. Traditional explanations for adhesion hysteresis, including viscoelasticity, capillarity, and plastic deformation, were ruled out using in situ observation of geometry and materials in the contact. 
Rather, variable work of adhesion due to stress-dependent covalent bond formation across the interface was confirmed and further elucidated by atomic-scale observation of the simulated contact and the increase in areal density of in-contact atoms.

\section{Acknowledgments}

The authors acknowledge support from the National Science Foundation under awards CMMI1536800 and CMMI-1537613. AM and XH further acknowledge the support of the Air Force Office of Scientific Research (AFOSR) award No. FA9550-15-1-0256. 


\section{$\underline{\text { References }}$}

1. Chou, S.Y., Krauss, P.R., Renstrom, P.J.: Imprint of sub-25 nm vias and trenches in polymers. Appl. Phys. Lett. 67, 3114-3116 (1995)

2. $\quad$ Piner, R.D., Zhu, J., Xu, F., Hong, S., Mirkin, C.A.A.: "Dip-Pen” nanolithography. Science 283, 661-663 (1999)

3. Kim, T.-H., Chung, D.-Y., Ku, J., Song, I., Sul, S., Kim, D.-H., Cho, K.-S., Choi, B.L., Min Kim, J., Hwang, S., Kim, K.: Heterogeneous stacking of nanodot monolayers by dry pick-and-place transfer and its applications in quantum dot light-emitting diodes. Nat. Commun. 4, 2637 (2013)

4. Majumdar, A.: Scanning thermal microscopy. Annu. Rev. Mater. Sci. 29, 505-585 (1999)

5. Park, J.Y., Maier, S., Hendriksen, B., Salmeron, M.: Sensing current and forces with SPM. Mater. Today. 13, 38-45 (2010)

6. Rebeiz, G.M., Muldavin, J.B.: RF MEMS switches and switch circuits. IEEE Microw. Mag. 2, 59-71 (2001). https://doi.org/10.1109/6668.969936

7. Loh, O.Y., Espinosa, H.D.: Nanoelectromechanical contact switches. Nat. Nanotechnol. 7, 283-295 (2012). https://doi.org/10.1038/nnano.2012.40

8. Maugis, D.: Adhesion of spheres: The JKR-DMT transition using a Dugdale model. J. Colloid Interface Sci. 150, 243-269 (1992). https://doi.org/10.1016/0021-9797(92)90285$\mathrm{T}$

9. Hertz, H.: On the contact of elastic solids. J. Reine Angew. Math. 92, 110 (1881)

10. Derjaguin, B. V, Muller, V.M., Toporov, Y.P.: Effect of contact deformations on the adhesion of particles. J. Colloid Interface Sci. 53, 314-326 (1975)

11. Johnson, K.L., Kendall, K., Roberts, A.D.: Surface energy and the contact of elastic solids. Proc. R. Soc. Lond. A 324, 301-313 (1971)

12. Tabor, D.: Surface forces and surface interactions. J. Colloid Interface Sci. 58, 2-13 (1977)

13. Carpick, R.W., Ogletree, D.F., Salmeron, M.: A general equation for fitting contact area and friction vs load measurements. J. Colloid Interface Sci. 211, 395-400 (1999)

14. Piétrement, O., Troyon, M.: General equations describing elastic indentation depth and normal contact stiffness versus load. J. Colloid Interface Sci. 226, 166-171 (2000).

15. Dong, Y., Li, Q., Martini, A.: Molecular dynamics simulation of atomic friction: A review and guide. J. Vac. Sci. Technol., A 31, 030801 (2013)

16. Chandross, M., Lorenz, C.D., Stevens, M.J., Grest, G.S.: Simulations of nanotribology with realistic probe tip models. Langmuir 24, 1240-1246 (2008)

17. Brukman, M.J., Gao, G., Nemanich, R.J., Harrison, J.A.: Temperature dependence of single-asperity diamond-diamond friction elucidated using AFM and MD simulations. J. Phys. Chem. C. 112, 9358-9369 (2008)

18. Li, Q., Dong, Y., Perez, D., Martini, A., Carpick, R.W.: Speed dependence of atomic stick-slip friction in optimally matched experiments and molecular dynamics simulations. Phys. Rev. Lett. 106, 126101 (2011)

19. Vahdat, V., Ryan, K.E., Keating, P.L., Jiang, Y., Adiga, S.P., Schall, J.D., Turner, K.T., Harrison, J.A., Carpick, R.W.: Atomic-scale wear of amorphous hydrogenated carbon during intermittent contact: A combined study using experiment, simulation, and theory. ACS Nano. 8, 7027-7040 (2014). https://doi.org/10.1021/nn501896e

20. Mo, Y., Szlufarska, I.: Roughness picture of friction in dry nanoscale contacts. Phys. Rev. 


\section{B. 81, 35405 (2010)}

21. Reedy, E.D.: Thin-coating contact mechanics with adhesion. J. Mater. Res. 21, 2660-2668 (2011)

22. Milne, Z.B., Schall, J.D., Jacobs, T.D.B., Harrison, J.A., Carpick, R.W.: Covalent bonding and atomic-level plasticity increase adhesion in silicon-diamond nanocontacts. Submitted. (2019)

23. Luan, B., Robbins, M.O.: The breakdown of continuum models for mechanical contacts. Nature 435, 929-932 (2005)

24. Luan, B.Q., Robbins, M.O.: Contact of single asperities with varying adhesion: Comparing continuum mechanics to atomistic simulations. Phys. Rev. E. 74, 026111 (2006). https://doi.org/10.1103/PhysRevE.74.026111

25. Cha, P.-R., Srolovitz, D.J., Vanderlick, T.K.: Molecular dynamics simulation of single asperity contact. Acta Mater. 55, 3983-3996 (2004)

26. Szlufarska, I., Chandross, M., Carpick, R.W.: Recent advances in single-asperity nanotribology. J. Phys. D: Appl. Phys. 41, 123001 (2008)

27. Ryan, K.E., Keating, P.L., Jacobs, T.D.B., Grierson, D.S., Turner, K.T., Carpick, R.W., Harrison, J.A.: Simulated adhesion between realistic hydrocarbon materials: Effects of composition, roughness, and contact point. Langmuir 30, 2028-2037 (2014)

28. Mo, Y., Turner, K.T., Szlufarska, I.: Friction laws at the nanoscale. Nature 457, 11161119 (2009)

29. Solhjoo, S., Vakis, A.I.: Definition and detection of contact in atomistic simulations. Comput. Mater. Sci. 109, 172-182 (2015)

30. Jacobs, T.D.B., Martini, A.: Measuring and understanding contact area the nanoscale: A review. Appl. Mech. Rev. 69, 060802 (2017). https://doi.org/10.1115/1.4038130

31. Carpick, R.W., Agrait, N., Ogletree, D.F., Salmeron, M.: Variation of the interfacial shear strength and adhesion of a nanometer-sized contact. Langmuir 12, 3334-3340 (1996)

32. Enachescu, M., van den Oetelaar, R.J.A., Carpick, R.W., Ogletree, D.F., Flipse, C.F.J., Salmeron, M.: Atomic force microscopy study of an ideally hard contact: The diamond(111) tungsten carbide interface. Phys. Rev. Lett. 81, 1877-1880 (1998)

33. Carpick, R.W., Agrait, N., Ogletree, D.F., Salmeron, M.: Measurement of interfacial shear (friction) with an ultrahigh vacuum atomic force microscope. J. Vac. Sci. Technol. B: Microelectron. Nanometer Struct.--Process., Meas., Phenom. 14, 1289-1295 (1996)

34. Minor, A.M., Lilleodden, E.T., Jin, M., Stach, E.A., Chrzan, D.C., Morris, J.W.: Room temperature dislocation plasticity in silicon. Philos. Mag. 85, 323-330 (2005)

35. Unertl, W.N.: Implications of contact mechanics models for mechanical properties measurements using scanning force microscopy. J. Vac. Sci. Technol., A 17, 1779 (1999)

36. Espinosa, H.D., Bernal, R.A., Minary-Jolandan, M.: A review of mechanical and electromechanical properties of piezoelectric nanowires. Adv. Mater. 24, 46564675(2012). https://doi.org/10.1002/adma.201104810

37. Jacobs, T.D.B., Carpick, R.W.: Nanoscale wear as a stress-assisted chemical reaction. Nat. Nanotechnol. 8, 108-112 (2013). https://doi.org/10.1038/nnano.2012.255

38. Vishnubhotla, S.B., Chen, R., Khanal, S.R., Martini, A., Jacobs, T.D.B.: Understanding contact between platinum nanocontacts at low loads: The effect of reversible plasticity. Nanotechnology. 30, 035704 (2019). https://doi.org/10.1088/1361-6528/aaea2b

39. Vishnubhotla, S.B., Chen, R., Khanal, S.R., Li, J., Stach, E.A., Martini, A., Jacobs, T.D.B.: Quantitative measurement of contact area and electrical transport across platinum 
nanocontacts for scanning probe microscopy and electrical nanodevices. Nanotechnology. 30, 045705 (2019). https://doi.org/10.1088/1361-6528/aaebd6

40. Jacobs, T.D.B., Lefever, J.A., Carpick, R.W.: Measurement of the length and strength of adhesive interactions in a nanoscale silicon-diamond interface. Adv. Mater. Interfaces. 2, 1400547 (2015). https://doi.org/10.1002/admi.201400547

41. Williams, D.B., Carter, C.B.: The Transmission Electron Microscope. Springer, Boston (1996)

42. Kailer, A., Gogotsi, Y.G., Nickel, K.G.: Phase transformations of silicon caused by contact loading. J. Appl. Phys. 81, 3057-3063 (1997)

43. Liu, J., Notbohm, J.K., Carpick, R.W., Turner, K.T.: Method for characterizing nanoscale wear of atomic force microscope tips. ACS Nano. 4, 3763-3772 (2010).

https://doi.org/10.1021/nn100246g

44. Johnson, K.L.: Contact Mechanics. Cambridge University Press, London (2011)

45. Plimpton, S.J.: Fast parallel algorithms for short-range molecular dynamics. J. Comput. Phys. 117, 1-19 (1995). https://doi.org/10.1006/jcph.1995.1039

46. Kumagai, T., Izumi, S., Hara, S., Sakai, S.: Development of bond-order potentials that can reproduce the elastic constants and melting point of silicon for classical molecular dynamics simulation. Comput. Mater. Sci. 39, 457-464 (2007).

https://doi.org/10.1016/j.commatsci.2006.07.013

47. Guo, Q., Izumisawa, S., Phillips, D.M., Jhon, M.S.: Surface morphology and molecular conformation for ultrathin lubricant films with functional end groups. J. Appl. Phys. 93, 8707-8709 (2003)

48. Yi, T., Ramasamy, U.S., Lichter, S., Martini, A.: Stability and structure of nanometer-thin perfluoropolyether films using molecular simulations. Tribol. Lett. 54, 119-127 (2014)

49. Cottrell, T.L.: The Strengths of Chemical Bonds. Butterworth, London (1958)

50. Grierson, D.S., Flater, E.E., Carpick, R.W.: Accounting for the JKR-DMT transition in adhesion and friction measurements with atomic force microscopy. J. Adhes. Sci.

Technol. 19, 291-311 (2005)

51. Katainen, J., Paajanen, M., Ahtola, E., Pore, V., Lahtinen, J.: Adhesion as an interplay between particle size and surface roughness. J. Colloid Interface Sci. 304, 524-529 (2006)

52. Liu, D.L., Martin, J., Burnham, N.A.: Optimal roughness for minimal adhesion. Appl. Phys. Lett. 91, 043107 (2007)

53. Grierson, D.S., Liu, J., Carpick, R.W., Turner, K.T.: Adhesion of nanoscale asperities with power-law profiles. J. Mech. Phys. Solids. 61, 597-610 (2013)

54. Moore, N.W., Houston, J.E.: The pull-off force and the work of adhesion: New challenges at the nanoscale. J. Adhes. Sci. Technol. 24, 2531-2544 (2010)

55. Sumant, A. V, Grierson, D.S., Gerbi, J.E., Birrell, J., Lanke, U.D., Auciello, O., Carlisle, J.A., Carpick, R.W.: Toward the ultimate tribological interface: Surface chemistry and nanotribology of ultrananocrystalline diamond. Adv. Mater. 17, 1039-1045 (2005)

56. Sumant, A., Grierson, D., Gerbi, J., Carlisle, J., Auciello, O., Carpick, R.: Surface chemistry and bonding configuration of ultrananocrystalline diamond surfaces and their effects on nanotribological properties. Phys. Rev. B. 76, 235429 (2007)

57. Israelachvili, J.N.: Intermolecular and Surface forces (Third Edition). Elsevier, San Francisco (2010)

58. Burnham, N.A., Colton, R.J., Pollock, H.M.: Interpretation issues in force microscopy. J. Vac. Sci. Technol., A 9, 2548 (1991). https://doi.org/10.1116/1.577271 
59. Cheng, S., Robbins, M.O.: Defining contact at the atomic scale. Tribol. Lett. 39, 329-348 (2010)

60. Cheng, S., Luan, B., Robbins, M.O.: Contact and friction of nanoasperities: Effects of adsorbed monolayers. Phys. Rev. E. 81, 016102 (2010)

61. McSkimin, H.J.: Elastic moduli of diamond as a function of pressure and temperature. J. Appl. Phys. 43, 2944 (1972)

62. Hopcroft, M.A., Nix, W.D., Kenny, T.W.: What is the young's modulus of silicon? J. Microelectromech. Syst. 19, 229-238 (2010)

63. Chen, R., Vishnubhotla, S.B., Jacobs, T.D.B., Martini, A.: Simulations of the effect of an oxide on contact area measurements from conductive atomic force microscopy.

Nanoscale, 11, 1029-1036 (2019)

64. De Boer, M.P., Knapp, J.A., Michalske, T.A., Srinivasan, U., Maboudian, R.: Adhesion hysteresis of silane coated microcantilevers. Acta Mater. 48, 4531-4541 (2000). https://doi.org/10.1016/S1359-6454(00)00239-1

65. Attard, P.: Interaction and deformation of viscoelastic particles: Nonadhesive particles. Phys. Rev. E. 63, 61604 (2001)

66. Chen, Y.L., Helm, C.A., Israelachvili, J.N.: Molecular mechanisms associated with adhesion and contact angle hysteresis of monolayer surfaces. J. Phys. Chem. 95, 1073610747 (1991)

67. Horn, R.G., Israelachvili, J.N., Pribac, F.: Measurement of the deformation and adhesion of solids in contact. J. Colloid Interface Sci. 115, 480-492 (1987) 